\title{
Engineering, Characterization and Directional Self- Assembly of Anisotropically Modified Nanocolloids
}

\author{
Srijanani Bhaskar, Christopher T. Gibson, Mutsumi Yoshida, Himabindu Nandivada, \\ Xiaopei Deng, Nicolas H. Voelcker, and Joerg Lahann*
}

\begin{abstract}
Along with traditional attributes such as the size, shape, and chemical structure of polymeric micro-objects, control over material distribution, or selective compartmentalization, appears to be increasingly important for maximizing the functionality and efficacy of biomaterials. The fabrication of tri- and tetracompartmental colloids made from biodegradable poly(lactide-co-glycolide) polymers via electrohydrodynamic co-jetting is demonstrated. The presence of three compartments is confirmed via flow cytometry. Additional chemical functionality is introduced via the incorporation of acetylene-functionalized polymers into individual compartments of the particles. Direct visualization of the spatioselective distribution of acetylene groups is demonstrated by confocal Raman microscopy as well as by reaction of the acetylene groups with azide-biotin via 'click chemistry'. Biotinstreptavidin binding is then utilized for the controlled assembly and orientation of bicompartmental particles onto functionalized, micropatterned substrates prepared via chemical vapor deposition polymerization.
\end{abstract}

\section{Introduction}

Nano- and microparticles are the fundamental design elements in a variety of research areas, such as diagnostics, therapeutics, and photonic crystals. Progress in particle engineering has continuously increased their versatility and tunability in these applications. ${ }^{[1,2]}$ While a variety of techniques have been developed to precisely engineer nano- and microparticles with respect to shape, size, surface chemistry, and mechanical properties, ${ }^{[3]}$ control over anisotropic

Prof. J. Lahann

Departments of Chemical Engineering

Materials Science and Engineering

Macromolecular Science and Engineering and Biomedical Engineering

University of Michigan

Ann Arbor, 48109, USA

E-mail: lahann@umich.edu

Dr. C. T. Gibson, Prof. N. H. Voelcker

Centre for Nanoscale Science and Technology

School of Chemical and Physical Sciences

Flinders University

Adelaide, 5001, Australia

DOI: 10.1002/smll.201001695 bulk and surface architectures are emerging as additional design parameters. ${ }^{[3,4]}$ Anisotropic particles may enable the formation of complex hierarchical assemblies needed for a diverse array of applications such as photonic band gap materials, ${ }^{[5,6]}$ multiplexed bioassays, ${ }^{[7]}$ vehicles for multiple therapeutic modalities, ${ }^{[8]}$ biological sensors, biohybrid materials, ${ }^{[9,10]}$ and miniaturized energy storage and conversion devices. ${ }^{[11]}$ Synthetic processes ${ }^{[4,6,12-15]}$ for anisotropic particles include lithographic techniques, ${ }^{[16]}$ seeded polymerization, ${ }^{[17]}$ and self-assembly. ${ }^{[18]}$ We have previously

\author{
S. Bhaskar, X. Deng \\ Macromolecular Science and Engineering \\ University of Michigan \\ Ann Arbor, 48109, USA \\ Dr. M. Yoshida, Dr. H. Nandivada ${ }^{[+]}$ \\ Department of Chemical Engineering \\ University of Michigan \\ Ann Arbor, 48109, USA \\ [+] Current Address: BD Biosciences, Bedford, MA 01730, USA
}


used electrohydrodynamic co-jetting for the fabrication of anisotropic particles. ${ }^{[19]}$ In its simplest form, this process involves the coprocessing of two polymer solutions through a side-by-side dual capillary needle system under a laminar flow regime. Application of an electric field to the compound droplet generated at the tips of the capillary needles results in the distortion of the droplet into a Taylor cone and the formation of an electrified jet, from which bicompartmental particles are generated. We first demonstrated this process from aqueous solutions of poly(acrylic acid-co-acrylamide) polymers. ${ }^{[19-21]}$ More recently, we extended this process to include particles and fibers comprised of apolar and biodegradable polymers, namely poly(lactide-co-glycolide)s (PLGA). Here, organic solvents are used during electrohydrodynamic cojetting. ${ }^{22-24]}$ Systematic variation of the jetting solution and process parameters allows for control over shape and size. ${ }^{[25]}$ Apart from the precise control over particle characteristics, the potentially most salient advantage of the electrohydrodynamic co-jetting process may be the ability to fabricate particles with more than two compartments by simply increasing the number of capillaries from which the solutions are processed. ${ }^{24]}$ Here, we report on the selective surface modification of novel bi-, tri- and tetracompartmental particles for directed self-assembly.

\section{Results and Discussion}

\subsection{Synthesis of Anisotropic Microparticles}

Our previous attempts to fabricate microstructures from PLGA-containing organic solutions with more than two compartments targeted fibers and involved jetting solutions of significantly higher viscosities compared to those typically employed in the case of particles. ${ }^{[23,24]}$ This higher viscosity not only provides resistance towards jet breakup, but also imparts stability to the jet, resulting in extremely well-defined compartments. ${ }^{[23,24]}$ As a starting point, we employed three solutions, each containing $4.3 \mathrm{wt} \%$ PLGA in 97:3 volume ratio of chloroform and $N, N$ dimethyl formamide (DMF). To assist the visualization of the multicompartmental character of the particles, the jetting solutions further contained fluorophores with distinct emission characteristics. Specifically, we used poly[tris(2,5-bis(hexyloxy)1,4 - phenylenevinylene ) - alt - (1,3 phenylenevinylene) (PTDPV, green), as well as the polythiophene-based polymers ADS406PT (blue) and ADS306PT (red). These solutions were pumped through three capillary needles arranged in a triangular configuration. The flow rate was maintained at $0.15 \mathrm{~mL} \mathrm{~h}^{-1}$. Upon application of a DC voltage of $\sim 7.5 \mathrm{kV}$, the liquid droplet that formed at the tip of the capillaries was distorted into a single Taylor cone. ${ }^{[26]}$ Stretching of the compound droplet under the influence of the applied electric field resulted in an electrified jet, which produced particles as a result of solvent evaporation. Figure 1a shows confocal laser scanning microscopy (CLSM) images of the resulting tricompartmental microparticles, which were collected at the counterelectrode. Blue, green, and red channels are shown individually, followed by their overlay, which confirms the presence of remarkably distinct compartments with well-defined interfaces. Scanning electron microscopy (SEM) images (Supporting Information (SI), Figure S1) confirmed the spherical shape of the particles. The CLSM images shown in Figure 1a depict larger particles for the sake of clarity; however, further examination (SI, Figure S2) revealed clearly defined compartments in smaller particles as well. Encouraged by our ability to form tricompartmental particles, we extended this process to include four capillary needles arranged in a square configuration. In this case, we reduced the flow rate to $0.1 \mathrm{~mL} \mathrm{~h}^{-1}$ to maintain similar droplet volumes, which were processed with similar voltages. Because of the limited availability of suitable dyes with distinct fluorescence emission spectra, three solutions contained the ADS306PT dye and the fourth one with PTDPV. Jetting in the cone jet mode described above resulted in the formation of tetracompartmental particles, shown in Figure 1b, with a well-defined 'quarter' compartment which is green and a larger red compartment which is a composite of three remaining compartments. Interestingly, compared to fibers, ${ }^{[24]}$ isomeric needle arrangements, ${ }^{[24]}$ i.e, different ways of positioning a given number of capillaries in a side-by-side manner, did not produce any changes in the nature of compartmentalization (data not shown). For instance, similar particle geometries were obtained from striped versus triangular- or

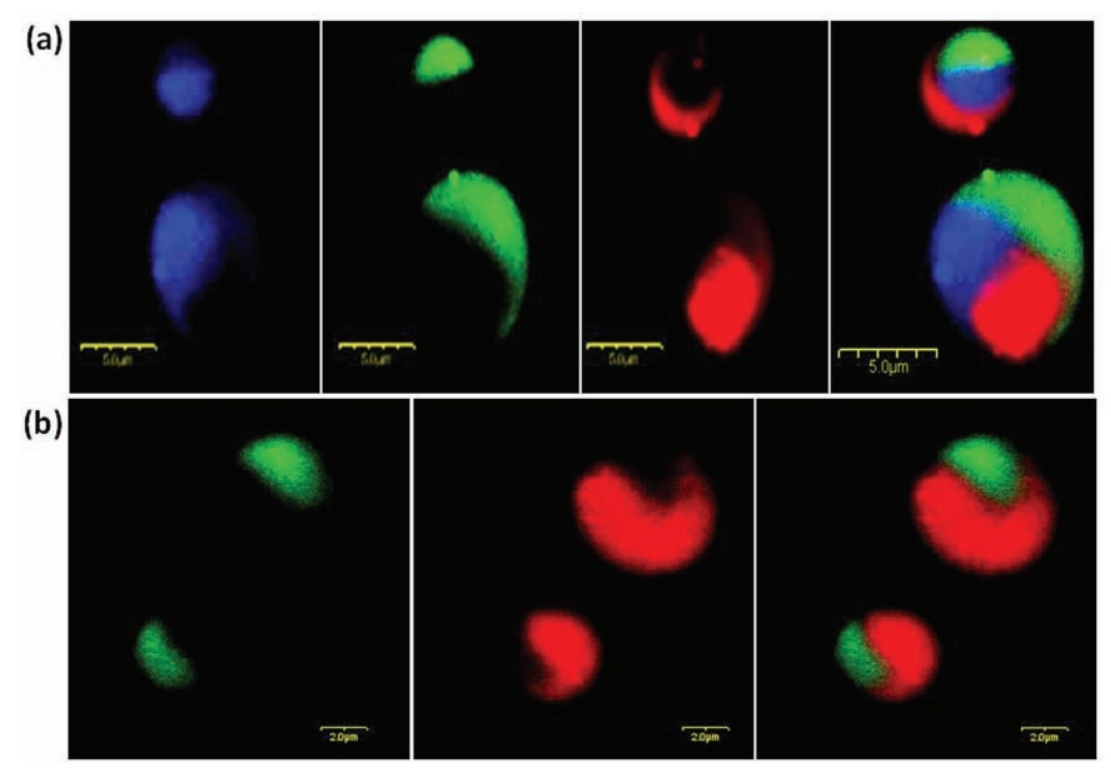

Figure 1. a) CLSM image of tricompartmental PLGA microparticles synthesized via electrohydrodynamic co-jetting from three capillaries in a triangular configuration. Individual fluorescence channels resulting from blue, green, and red dyes are shown, followed by the overlay. b) CLSM image of tetracompartmental microparticles made via co-jetting from four capillary needles arranged in a square configuration. Three solutions were labeled with ADS306PT (red) and the fourth solution was labeled with PTDPV (green). Scale bars represent $5 \mu \mathrm{m}$ and $2 \mu \mathrm{m}$ for (a) and (b), respectively. 
square-shaped needle arrangements for tri- and tetracompartmental particles, respectively. This is distinctly different from multicompartmental fibers ${ }^{[24]}$ where the arrangement of the jetting needle influences the fiber architecture. A reason for the difference could be the lower viscosity of the jetting solutions, which can cause swirling motions in the Taylor cone, ${ }^{[19]}$ ultimately impeding the formation of precise architectures. A related aspect is the uniformity of individual compartments over a large population of particles. Indeed, examination of a larger population of tricompartmental particles revealed a variety of compartmental arrangements (SI, Figure S2), echoing a trend previously reported for bicompartmental particles, where "sandwich"-shaped as well as near-perfect side-by-side hemispheres were obtained. ${ }^{[22]}$ In contrast, monodisperse particles with close to perfectly uniform internal compartmentalization can be achieved by microsectioning of multicompartmenal fibers made by electrohydrodynamic co-jetting. ${ }^{[23]}$

\subsection{Characterization of Microparticle Compartmentalization}

In order to quantitatively assess the degree of compartmentalization, flow cytometry was used to analyze a typical population of tricompartmental particles. First, a negative control group of particles without any fluorescent dyes was analyzed. The fluorescence emission observed from this population was designated as background signal, which is depicted by a vertical line in each of the three plots shown in Figure 2a. Next, tricompartmental particles were fabricated, but loaded with only one fluorescent dye per group. Each of these populations exhibited an increase in fluorescence intensity corresponding to the representative channel (Figures 2b,c,d). For instance, tricompartmental particles loaded with ADS406PT dye exhibited an increase in fluorescence intensity in the blue channel (Figure 2b). Moreover, the fluorescence intensities of the other channels (here: green and red) were still below the background threshold level, indicating negligible bleed-through of spectral emission. This was further confirmed using particles that were loaded with different dyes in two out of three compartments. The fluorescence histograms of these particles also presented a similar trend, i.e., an increase in fluorescence intensity in the channels corresponding to the two respective dyes (Figures 2e,f,g). Finally, tricompartmental particles containing blue, green, and red dyes were analyzed. A significant increase in fluorescence intensity in all three channels was observed, confirming the tricompartmental nature of the particles (Figure $2 \mathrm{~h}$ ). For a population of 10000 particles, at least $80 \%$ of the particles were positive for blue, green, and red fluorescence (SI, Figure S3).
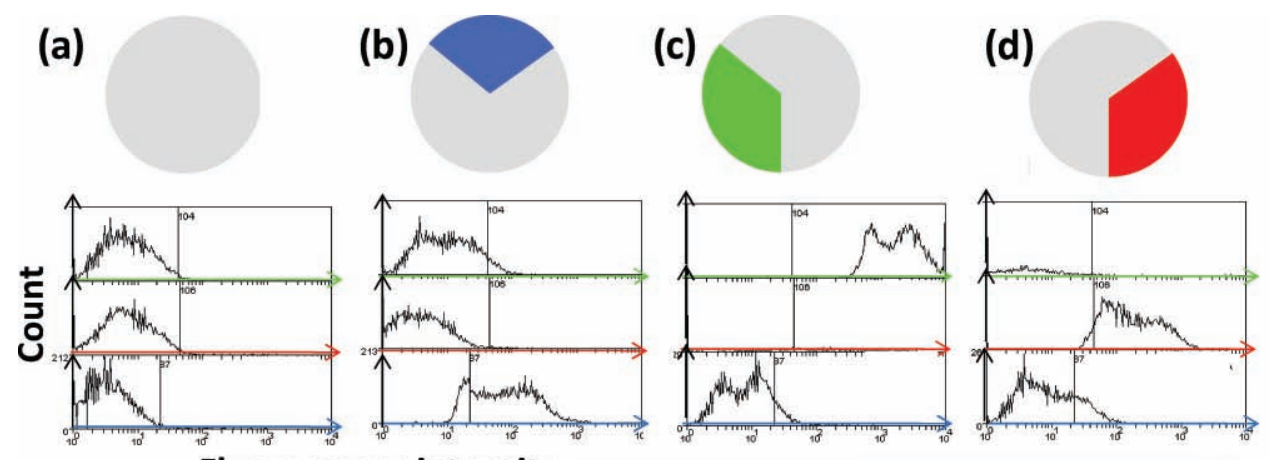

Fluorescence intensity
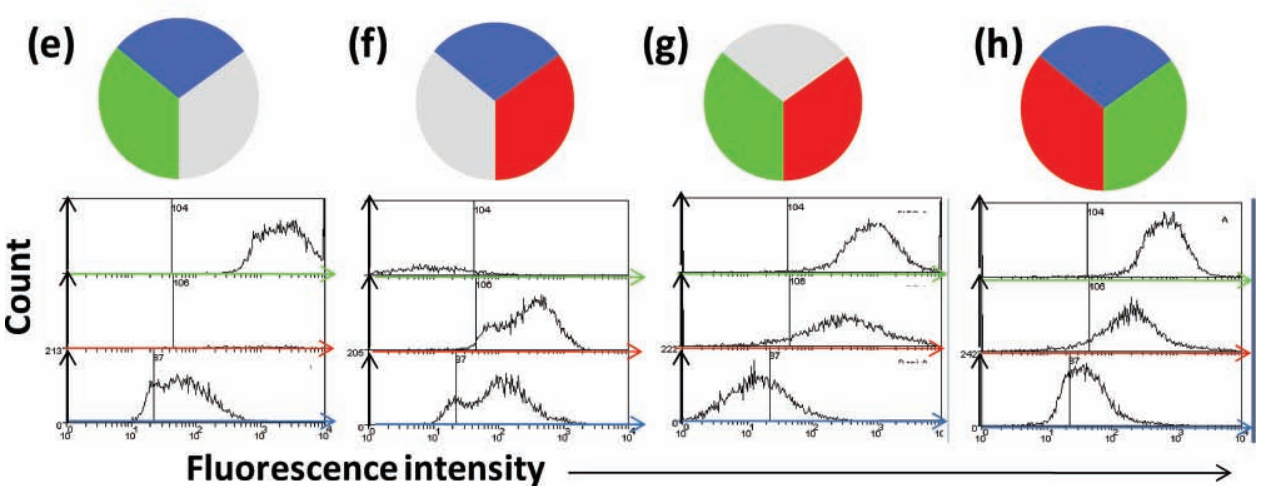

Figure 2. Flow cytometry analysis of tricompartmental particles. Each plot comprises an overlay of three histograms with a corresponding symbol indicating the nature of the dye-loading in the particles. For each plot, the $y$-axis represents particle count, and the $x$-axes denote the magnitude of fluorescence intensity from the dyes. Particles without any dye (a) were employed as a reference to determine background fluorescence. Particles with one dye $(b-d)$ and different permutations of two dyes $(e-g)$ were then analyzed. Particles loaded with one dye only exhibited a fluorescence intensity shift corresponding to that dye, and particles with two dyes showed significant intensity shifts corresponding to two dyes. Finally, tricompartmental particles containing three dyes (h) were analyzed and found to exhibit shifts in all three channels, confirming the tricompartmental nature of the particles. 


\subsection{Chemical Functionalization}

After the fabrication and characterization of multicompartmental PLGA particles, we sought to selectively introduce chemical functionality, the essential requirement for spatially directed self-assembly. Towards this end, we employed an acetylene-functionalized derivate of PLGA, polylactideco-poly(propargyl glycolide) (PLA-co-PPGL). ${ }^{[22,27]}$ During electrohydrodynamic co-jetting, PLGA blended with $23 \mathrm{wt} \%$ PLA-co-PPGL was used as one of the jetting solutions, while the other solution(s) were comprised of PLGA only. All other parameters were identical. In order to confirm selective compartmentalization of the acetylene-containing particles, we colocalized the polymer with a fluorescent dye and used CLSM to confirm the multicompartmental character of the particles (SI, Figure S4). While the images suggest compartmentalization, this approach has two potential disadvantages: 1) Because the fluorescence dye and PLA-co-PPGL are not covalently linked to the polymer, tracking the dyes is an indirect approach that assumes identical distribution of polymer and dye throughout the particle. 2) The addition of dyes increases the complexity of the process. Thus, direct visualization of the PLA-co-PPGL polymer without the use of dyes would be desirable. We therefore evaluated the use of confocal Raman spectromicroscopy (CRS) for direct characterization of PLA-co-PPGL distributions in bicompartmental particles. CRS has rapidly evolved as a powerful tool to characterize a diverse array of materials including drug-eluting coatings, ${ }^{[28]}$ the photopolymerisation of diacetylene gels, ${ }^{[29]}$ or the morphology of transition metal nanoparticulates. ${ }^{[30]}$ It provides spatially resolved physical and chemical information about materials directly in their native state without the need for external markers, such as fluorescent dyes or radioactive labels. CRS has the potential to be particularly well-suited to the analysis of multicompartmental particles, because the confocal scanning mode facilitates highresolution imaging, a feature required for imaging individual particles. ${ }^{[31]}$ The acetylene groups of PLA-co-PPGL are associated with a strong Raman band at 2120-2100 $\mathrm{cm}^{-1}$. ${ }^{[32]}$ This group was therefore used as a Raman label for mapping the vibrational signature of the acetylene groups within different areas of an isolated particle. Particles containing free acetylene groups in one compartment, but no fluorescent dyes, were prepared and imaged by CRS. Figure 3a,b show overlaid CRS maps of bicompartmental particles, where the PLA-co-PPGL comprised $50 \%$ and $25 \%$ of the particle, respectively. The red-colored maps correspond to the

(c) (b), respectively. intensity of the band at $1767 \mathrm{~cm}^{-1}$, which can be attributed to the ester groups of PLGA, ${ }^{[31]}$ and which is present throughout the particles. The yellow-colored maps show the intensity of the signal at $2122 \mathrm{~cm}^{-1}$. As mentioned above, this signal represents the carbon-carbon triple bond stretch indicative of the acetylene groups. The focal plane for the Raman spectral maps was located approximately at the midpoint of each particle. Figure $3 c$ shows representative Raman spectra from the particle shown in Figure 3a. The position of the red spectral trace is denoted by the + and the position of the yellow spectrum by the $\mathrm{x}$. The acetylene band can be clearly observed in Raman spectra of the yellow region of the particle image. In contrast, this band is absent in spectra of the red region of the particle. This confirms that the remaining volume of the particle is void of acetylene groups.

\subsection{Demonstration of Surface Activity}

After establishing the selective compartmentalization of acetylene groups in bicompartmental particles, we proceeded to demonstrate their surface activity by selectively

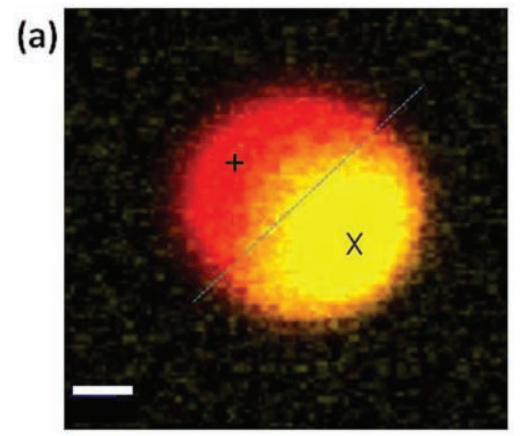

(b)
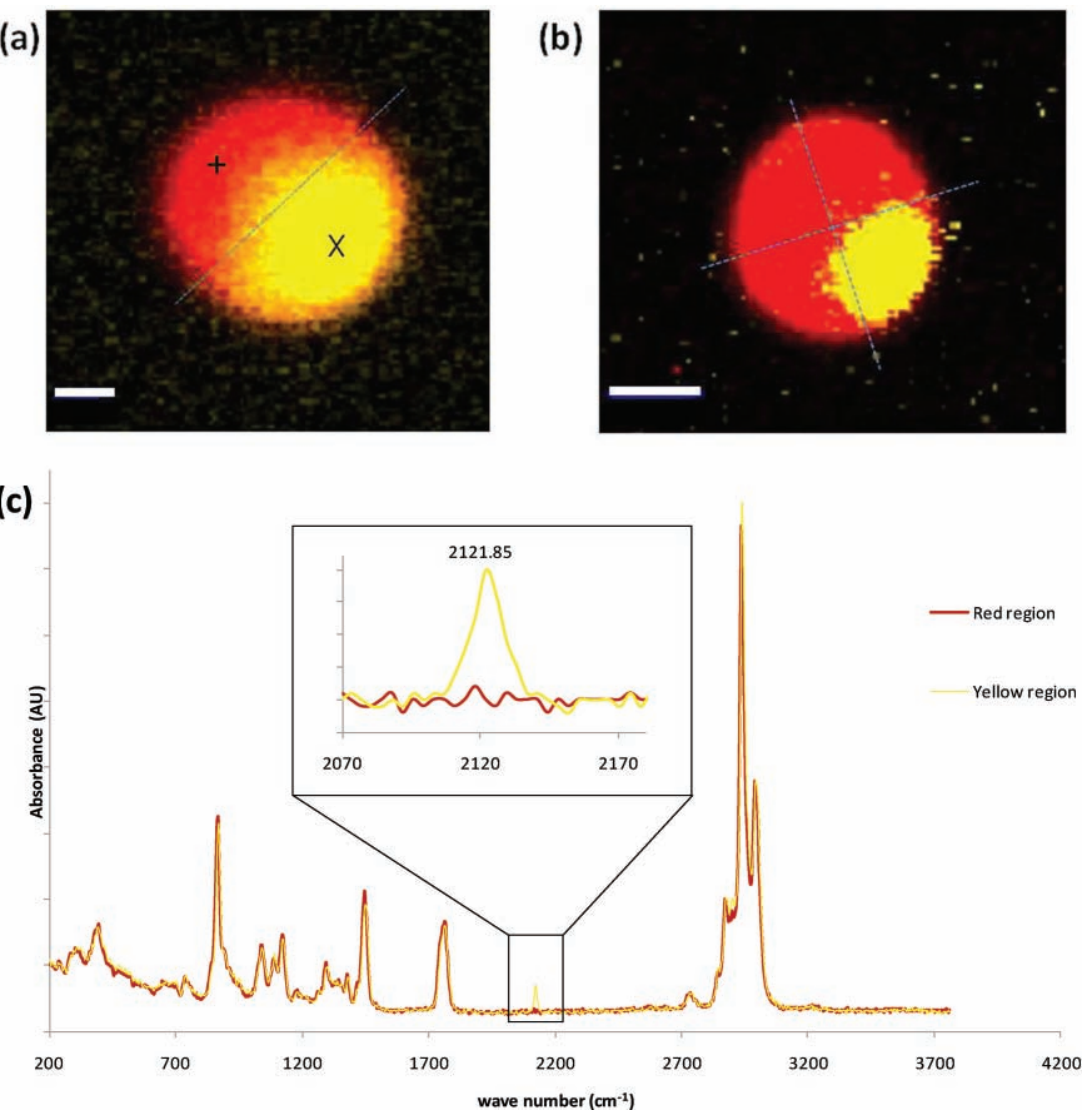

Figure 3. a) Raman image of a bicompartmental particle comprising a 30:100 w/w ratio of PLGA:PLA-co-PPGL in one compartment only. b) Raman-CLSM image of a tetracompartmental particle loaded with 23 wt\% PPGL in one out of four compartments. Dashed blue lines are to guide the eye. c) Raman spectra of biphasic particles. Red and yellow spectra correspond to the $\boldsymbol{+}$ and $\mathbf{x}$ positions in (a), respectively. The peak corresponding to the acetylene functional group is absent in the red spectrum, but visible in the yellow spectrum, as highlighted in the magnified spectrum shown in the inset. Scale bars denote $1 \mu \mathrm{m}$ and $2 \mu \mathrm{m}$ for (a) and 
introducing biotin groups onto the particle surfaces through the Huisgen 1,3-dipolar cycloaddition reaction between azides and terminal alkynes, widely known as 'click' chemistry. ${ }^{[3-35]}$ Owing to its stability, orthogonality, efficiency, and ability to proceed under physiological conditions, this set of reactions are being extensively applied towards the design of functional nanomaterials and polymers for pharmaceutical and biomedical applications. ${ }^{[34,36-38]}$ Bicompartmental particles containing PLA-co-PPGL and PLGA compartments labeled with PTDPV and ADS306 dyes were reacted with azide-poly(ethylene oxide)-biotin (azide-PEO-biotin) in the presence of $\mathrm{Cu}^{+}$ions generated by the reduction of $\mathrm{CuSO}_{4} \cdot 5 \mathrm{H}_{2} \mathrm{O}$ via sodium ascorbate. This resulted in the immobilization of biotin-azide on the surface of one compartment only. In order to confirm the selectivity of the click reaction, we incubated the biotinylated particles with Alexa Fluor 633-labeled streptavidin. This is depicted schematically in Figure 4a. The fluorescence from streptavidin (magenta) is clearly confined to one compartment only, as shown in Figure $4 \mathrm{~b}, \mathrm{c}$ for bicompartmental particles with $50 \%$ and $25 \%$ acetylene coverage, respectively. Fluorescence on one

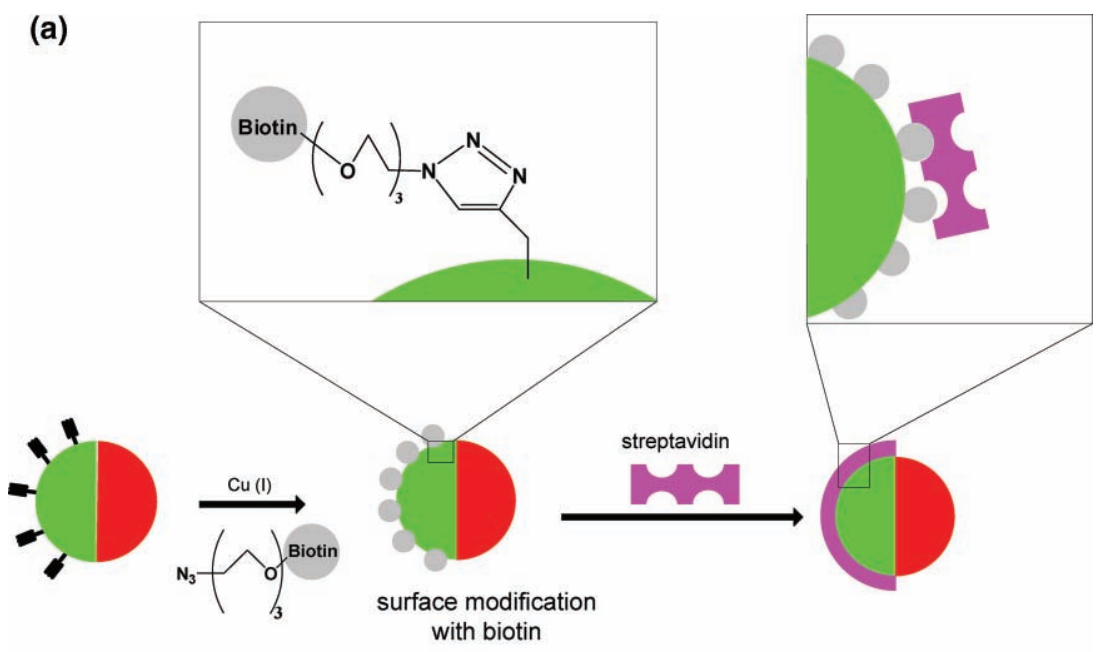

(b)

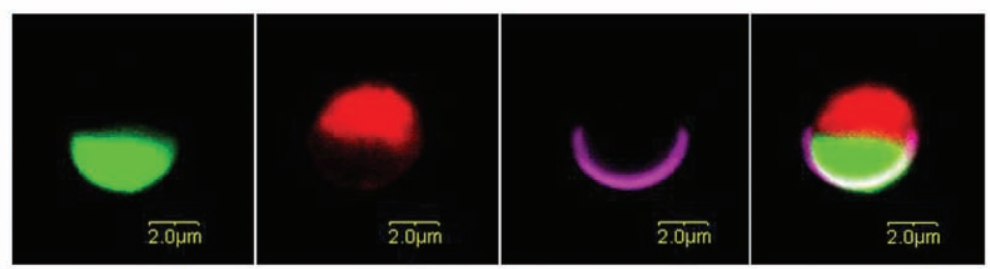

(c)

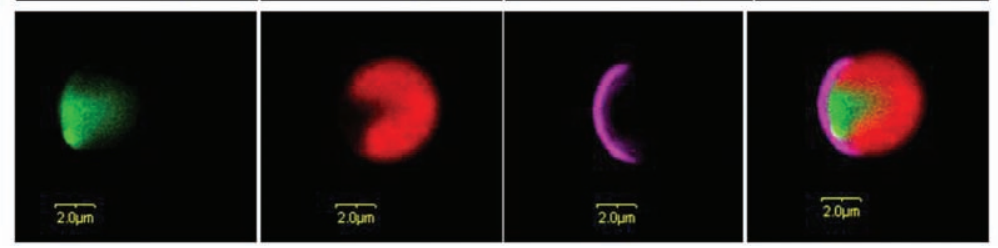

Figure 4. a) Schematic of surface modification of bicompartmental particles containing acetylene groups in one compartment only with azide-PEO-biotin. The ability of the particles to selectively bind with biotin is verified via incubation with Alexa Fluor 633-labeled streptavidin, resulting in spatioselective fluorescence. $b, c)$ CLSM images of bi- and tetracompartmental particles surface-modified with biotin on one compartment only. Green, red, and magenta channels (indicating fluorescence from PTDPV, ADS306PT and Alexa Fluor 633-streptavidin) are shown, followed by the overlay, which confirms spatioselective presence of streptavidin, and therefore biotin. compartment is visible in a majority of the population of particles, thereby confirming the homogenous distribution of acetylene fuctionality in the compartment containing PLAco-PPGL uniformity, the selectivity of the click reaction (SI, Figure S4) and the absence of nonspecific adsorption of streptavidin.

\subsection{Self-Assembly of Bicompartmental Particles}

Once the ability of bicompartmental particles to undergo spatioselective chemical modification was demonstrated, we investigated self-assembly onto patterned surfaces. Several methods have been employed to generate patterns of isotropic nanoparticles on surfaces, such as dip-pen lithography, ${ }^{[39]}$ patterning oligonucleotides via electron beam writing, ${ }^{[40]}$ Maragoni-flow induced patterning of colloids, ${ }^{[41]}$ and capillary flow of particles on top of patterned substrates. ${ }^{[18]}$ Patterning bicompartmental particles introduces an additional parameter: the selective addressability of the surface of one hemisphere. The other hemisphere is left free to react in an independent manner, for example, allowing orthogonal detection/ recognition of analytes, which is not possible without the spatial confinement of functional groups due to compartmentalization. Micropatterned streptavidinpresenting substrates were incubated with particles as depicted schematically in Figure 5a. Monocompartmental particles with biotin groups were first incubated with patterned streptavidin surfaces. CLSM imaging of surfaces showed that particles were present only in areas containing streptavidin (SI, Figure S5). Additionally, a particle population that was not reacted with biotin was tested as the control group. These particles did not form patterns on the substrate (SI, Figure S5), indicating that particle self-assembly was due to specific biotin-streptavidin interactions, and not nonspecific adsorption. The surfaces were then incubated with bicompartmental particles containing green and red dyes. These particles had biotin groups on the surface of the green compartment only. Patterns formed by these particles are shown in Figure 5b. Particle orientation is clear in the high-magnification images (Figure 5c), where the red hemisphere is more apparent on the surface in comparison with the green. Randomly oriented particles were also observed in certain areas (SI, Figure S6), where both red and green hemispheres could be visualized on the surface in the same focal plane. In these areas, nonspecific adhesion between the particles and the substrate occured, attributed to steric crowding of particles. 


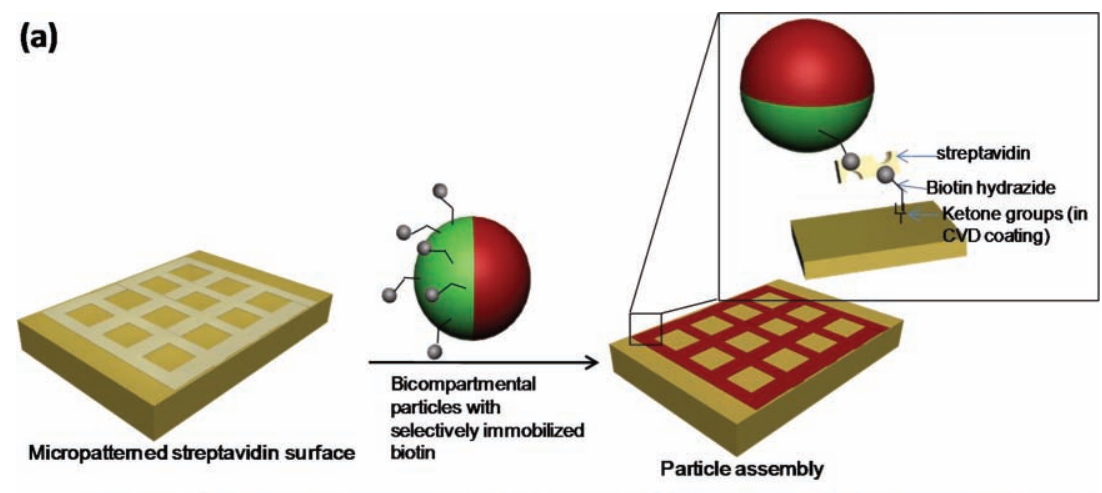

(b)

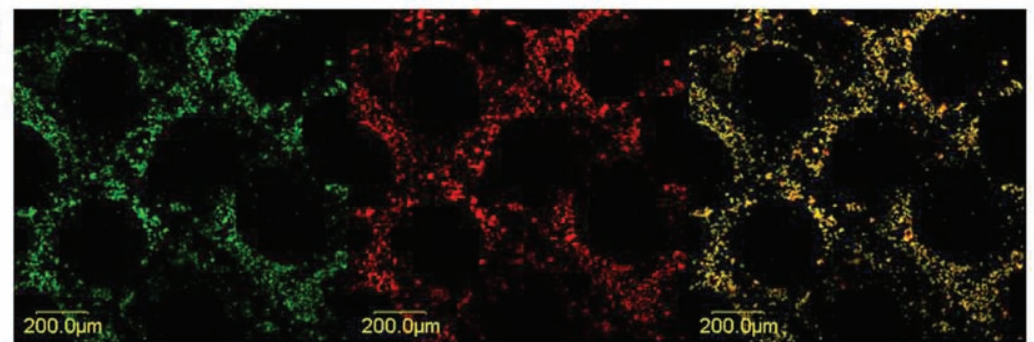

(c)

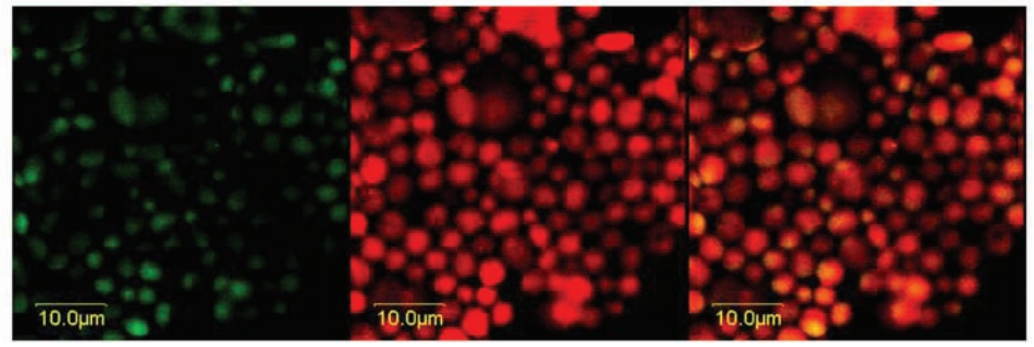

Figure 5. a) Scheme depicting the assembly of bicompartmental particles onto patterned streptavidin surfaces. A reactive chemical vapor deposition (CVD)-based coating containing ketone functional groups is deposited on silica surfaces. This is followed by microcontact printing of biotin-hydrazide, followed by immobilization of streptavidin to yield patterned substrates. Bicompartmental particles containing surface biotin groups on the green compartment then spontaneously assemble on these substrates, and selective binding of the green half leads to particle orientation. b) CLSM image of particles as seen on the streptavidin substrates. c) High-magnification CLSM image showing particle orientation.

\section{Conclusion}

In summary, we have extended the electrohydrodynamic co-jetting process to include composite flow from three and four capillary needles, resulting in formation of more complex internal particle architectures in the form of tri- and tetracompartmental particles. We have further introduced selective chemical functionality throughout the bulk of the bi- and tetracompartmental particles by incorporating an acetylenefunctionalized PLGA derivative during the electrohydrodynamic co-jetting process. The acetylene groups can be used for direct visualization by Raman microscopy. In addition, we have shown selective chemical activity by modifying half (or one quarter) of the particle surfaces with biotin, and assembling them on streptavidin-presenting substrates with a fair degree of spatial orientation. Future work will be directed towards conducting orthogonal chemical reactions on particles while attached to a substrate. Similar multicompartmental particles may find application in analyte recognition in diagnostic tests, in energy storage devices, or as biohybrid materials.

\section{Experimental Section}

Materials: Polylactide-co-glycolide with a lactide:slycolide ratio of 85:15 $\left(M_{\mathrm{w}} 40000-\right.$ $\left.75000 \mathrm{~g} \mathrm{~mol}^{-1}\right)$, poly[tris(2,5-bis(hexyloxy)1,4-phenylenevinylene)-alt-(1,3-phenylenevinylene) (PTDPV), chloroform, $N, N$ dimethylformamide (DMF), serum bovine albumin (BSA), fetal bovine serum (FBS), Tween-20, copper sulfate pentahydrate $\left(\mathrm{CuSO}_{4} \cdot 5 \mathrm{H}_{2} \mathrm{O}\right)$, sodium ascorbate and phosphate buffered saline (PBS) were purchased from Sigma-Aldrich, USA. A Sylgard elastomer kit for fabricating poly(dimethyl siloxane) (PDMS) stamps was procured from Dow Corning, USA. Polythiophene polymers, sold under commercial names ADS306PT $\left(M_{\mathrm{w}} 20000-70000 \mathrm{~g} \mathrm{~mol}^{-1}\right)$ ADS406PT $\left(M_{\mathrm{w}}\right.$ $30000-80000 \mathrm{~g} \mathrm{~mol}^{-1}$ ) were purchased from American Dye Source, Canada. Tetrafluorophenylazide-(triethylene glycol)-biotin (azide-PEObiotin) and biotin hydrazide were purchased from Pierce Protein Research Products, Thermo Fisher Scientific, USA. Streptavidin and Alexa Fluor 633-labeled streptavidin were obtained from Invitrogen, USA.

Electrohydrodynamic Co-jetting: Electrohydrodynamic co-jetting was carried out in accordance with a previously reported protocol. ${ }^{[22]}$ Briefly, for tricompartmental particles, the three jetting solutions were pumped through a modified triple-capillary system (steel capillary diameter: 26 gauge, length: $8.2 \mathrm{~cm}$ ) held together in a triangular configuration via a thin piece of plastic tubing. The capillaries were connected to the cathode of a DC voltage source (Gamma High Voltage Research, USA) and the flow rate was controlled via a syringe pump (Kd Scientific, USA). It is noteworthy that the reported flow rates correspond to the setting on the pump, and due to flow from multiple capillaries, the actual flow rate equals the product of the value assigned to the pump and the number of capillaries. A flat piece of aluminum foil was used as a counterelectrode, which acted as the substrate for harvesting of particles. The distance between the capillary tip and the substrate was maintained in the range of 28-33 cm. All experiments were performed inside a fumehood at room temperature $\left(23^{\circ} \mathrm{C}\right)$. In case of tetracompartmental particles, four capillaries were held together in a square configuration.

Flow Cytometry: A filtered, $2.5 \mathrm{mg} \mathrm{mL}^{-1}$ suspension of particles (loaded with the desired permutations of ADS306PT, ADS406PT and PTDPV) was suspended in PBS and analyzed using a FACSVantage cell sorter (BD Biosciences, 3-laser: 488, 351, and $633 \mathrm{~nm}$ ). ADS406PT was excited using the $351 \mathrm{~nm}$ laser whereas PTDPV and ADS306PT were excited using the $488 \mathrm{~nm}$ laser. Dichroic filters of emission wavelengths $424 \pm 44,530 \pm 30$, and $585 \pm 47 \mathrm{~nm}$ were used to collect the fluorescence emission from ADS406PT, PTDPV, and ADS306PT. Data acquisition and analysis were performed using CellQuest Pro (BD Biosciences). 
Confocal Laser Scanning Microscopy: An Olympus Fluoview1000 CLSM was used to analyze particles in an aqueous suspension or jetted directly on top of glass coverslips which were placed on the aluminum substrate during electrohydrodynamic co-jetting. ADS406PT, PTDPV, and ADS306PT and Alexa-Fluor 633-labeled streptavidin were excited by $405 \mathrm{~nm}$ UV, $488 \mathrm{~nm}$ Argon, 533 nm Helium-Neon green, and 633 nm Helium-Neon Red lasers, respectively. Optical filters of emission wavelength $430-460,505-525$, and 560-600 nm were used to visualize the fluorescence of ADS406PT, PTDPV, and ADS306PT, respectively. A $660 \mathrm{~nm}$ long-pass filter was used to visualize fluorescence from Alexa-Fluor 633-labeled streptavidin.

Confocal Raman Spectromicroscopy (CRS): Raman spectra and images were collected with a WiTEC alpha300R Microscope in confocal imaging Raman mode using 40× (Numerical Aperture 0.6) objective with a $532 \mathrm{~nm} \mathrm{Nd-YAG}$ green $(E=2.33 \mathrm{eV})$ laser operating at constant power for each experiment up to a possible maximum of approximately $60 \mathrm{~mW}$. The sample surface was held perpendicular to the excitation source. Spectral images were acquired using an integration time of $0.5 \mathrm{~s}$ per pixel with each image composed of 100 pixels $\times 100$ pixels. Each pixel corresponds to a separate Raman spectrum, allowing thousands of spectra to be acquired during an image scan. Raman data was collected by the WiTEC Control software and analysed in the WiTEC Project software.

Selective Surface Functionalization of Particles: Bicompartmental particles containing acetylene groups in one compartment only were harvested from the aluminum substrate and suspended in deionized (DI) water containing 0.01 vol\% Tween-20. To $20 \mathrm{~mL}$ of a $0.5 \mathrm{mg} \mathrm{mL}^{-1}$ suspension, $1.4 \mathrm{~mL}$ of $1 \mathrm{mg} \mathrm{mL}^{-1}$ solution of azide-PEO-biotin was added, followed by aqueous $\mathrm{CuSO}_{4} \cdot 5 \mathrm{H}_{2} \mathrm{O}$ solution $(0.2 \mathrm{~mL}, 0.1 \mathrm{~m})$. Finally, sodium ascorbate $(0.2 \mathrm{~mL}, 1 \mathrm{~m})$ solution was added, and the reaction was allowed to occur under magnetic stirring for $15 \mathrm{~h}$ at room temperature. In case of tetracompartmental particles, the amounts of all reagents were scaled by a factor of half. Particles were then washed by addition of $20 \mathrm{~mL}$ DI water (containing 2 vol\% Tween-20), vortexing, centrifugation and aspiration of the supernatant. This process was repeated 10-12 times. Particles were then resuspended in PBS buffer $(\mathrm{pH}$ 7.4) consisting of $0.1 \%(\mathrm{w} / \mathrm{v}) \mathrm{BSA}$ and $0.02 \%(\mathrm{v} / \mathrm{v})$ Tween-20.

Assembly of Particles on CVD Substrates: A conformal coating of poly(4-heptadecafluorononanoyl-p-xylylene-co-p-xylylene) was deposited on a $3 \mathrm{~cm} \times 3 \mathrm{~cm}$ silica substrates via CVD polymerization of 4-heptadecafluorononanoyl[2,2]-paracyclophane. ${ }^{[42]}$ This was followed by immobilization of biotin hydrazide via microcontact printing, wherein a poly(dimethylsiloxane) (PDMS) stamp consisting of square-shaped indentations $\left(400 \mu \mathrm{m}^{2}\right)$ with $50 \mu \mathrm{m}$ gaps between the square edges. ${ }^{[43]}$ The patterned substrate was incubated for $2 \mathrm{~h}$ with streptavidin or Alexa Fluor 633-labeled streptavidin in PBS buffer $(\mathrm{pH} 7.4)$ consisting of BSA $(0.1 \% \mathrm{w} / \mathrm{v})$ and Tween-20 (0.02 vol\%). The substrate was washed three times with the incubating buffer and rinsed with DI water. These substrates were then incubated with $100 \mu \mathrm{L}$ of an $8 \mathrm{mg} \mathrm{mL}^{-1}$ suspension of selectively biotinylated bicompartmental in PBS (containing $2 \%$ FBS and $1 \%$ Tween-20) at concentration for $2 \mathrm{~h}$, followed by gentle rinsing with $2 \%$ Tween-20 in DI water. For each case, control particles without any immobilized biotin were also incubated with streptavidin-presenting substrates in parallel. The surfaces were then imaged via CLSM.

\section{Supporting Information}

Supporting Information is available from the Wiley Online Library or from the author.

\section{Acknowledgements}

We thank Dr. Xuwei Jiang for synthesizing the PLA-Co-PPGL. This work has been supported by a grant from the American Cancer Society (RSG-08-284-01-CDD). S.B. and H.N. acknowledge financial support from Rackham Predoctoral Fellowship. Confocal Raman microscopy analysis was supported by the Australian Microscopy and Microanalysis Research Facility (AMMRF).

[1] M. Grzelczak, J. Vermant, E. M. Furst, L. M. Liz-Marzan, ACS Nano 2010, 4, 3591.

[2] S. Srivastava, A. Santos, K. Critchley, K. S. Kim, P. Podsiadlo, K. Sun, J. Lee, C. L. Xu, G. D. Lilly, S. C. Glotzer, N. A. Kotov, Science 2010, 327, 1355.

[3] S. Mitragotri, J. Lahann, Nat. Mater. 2009, 8, 15.

[4] S. Jiang, Q. Chen, M. Tripathy, E. Luijten, K. S. Schweizer, S. Granick, Adv. Mater. 2010, 22, 1060.

[5] A. M. Smith, S. Nie, Acc. Chem. Res. 2009, 43, 190.

[6] S. M. Yang, S. H. Kim, J. M. Lima, G. R. Yi, J. Mater. Chem. 2008, $18,2161$.

[7] D. C. Pregibon, M. Toner, P. S. Doyle, Science 2007, 315, 1393.

[8] S. Sengupta, D. Eavarone, I. Capila, G. Zhao, N. Watson, T. Kiziltepe, R. Sasisekharan, Nature 2005, 436, 568.

[9] M. Yoshida, K.-H. Roh, S. Mandal, S. Bhaskar, D. Lim, H. Nandivada, X. Deng, J. Lahann, Adv. Mater. 2009, 21, 4920.

[10] A. J. Swiston, J. B. Gilbert, D. J. Irvine, R. E. Cohen, M. F. Rubner, Biomacromolecules 2010, 11, 1826.

[11] G. J. Snyder, E. S. Toberer, Nat. Mater. 2008, 7, 105.

[12] K. J. Lee, J. Yoon, J. Lahann, Curr. Opin. Coll. Int. Sci. 2010, unpublished.

[13] A. Walther, A. H. E. Muller, Soft Matter 2008, 4, 663.

[14] M. Yoshida, J. Lahann, ACS Nano 2008, 2, 1101.

[15] F. Wurm, A. F. M. Kilbinger, Angew. Chem. Int. Ed. 2009, 48, 8412.

[16] D. Dendukuri, D. C. Pregibon, J. Collins, T. A. Hatton, P. S. Doyle, Nat. Mater. 2006, 5, 365.

[17] J. W. Kim, R. J. Larsen, D. A. Weitz, J. Am. Chem. Soc. 2006, 128, 14374.

[18] Y. N. Xia, Y. D. Yin, Y. Lu, J. McLellan, Adv. Funct. Mater. 2003, 13, 907.

[19] K. H. Roh, D. C. Martin, J. Lahann, Nat. Mater. 2005, 4, 759.

[20] K. H. Roh, D. C. Martin, J. Lahann, J. Am. Chem. Soc. 2006, 128, 6796.

[21] K. H. Roh, M. Yoshida, J. Lahann, Langmuir 2007, 23, 5683.

[22] S. Bhaskar, K. H. Roh, X. Jiang, G. L. Baker, J. Lahann, Macromol. Rapid Comm. 2008, 29, 1655.

[23] S. Bhaskar, J. Hitt, S. L. Chang, J. Lahann, Angew. Chem. Int. Ed. 2009, 48, 4589.

[24] S. Bhaskar, J. Lahann, J. Am. Chem. Soc. 2009, 131, 6650.

[25] S. Bhaskar, K. M. Pollock, M. Yoshida, J. Lahann, Small 2010, 6, 404.

[26] M. Cloupeau, B. Prunet-Foch, J. Electrostat. 1989, 22, 135.

[27] X. Jiang, E. B. Vogel, M. R. Smith III, G. L. Baker, Macromolecules 2008, 41, 1937. 
[28] A. Belu, C. Mahoney, K. Wormuth, J. Control. Release 2008, 126, 111.

[29] V. Haridas, Y. K. Sharma, R. Creasey, S. Sahu, C. Gibson, N. H. Voelcker, New J. Chem., DOI: 10.1039/C0NJ00544D.

[30] E. U. Donev, R. Lopez, L. C. Feldman, R. F. Haglund, Nano Lett. 2009, 9, 702.

[31] A. A. v. Apeldoorn, H. J. v. Manen, J. M. Bezemer, J. D. d. Bruijn, C. A. v. Blitterswijk, C. Otto, J. Am. Chem. Soc. 2004, 126, 13226.

[32] D. Lin-Vien, N. B. Colthup, W. G. Fateley, J. G. Grasselli, The Handbook of Infrared and Raman Characteristic Frequencies of Organic Molecules, Academic Press, San Diego, 1991.

[33] H. Nandivada, H. Y. Chen, L. Bondarenko, J. Lahann, Angew. Chem. Int. Ed. 2006, 45, 3360.

[34] H. Nandivada, X. Jiang, J. Lahann, Adv Mater. 2007, 19, 2197.

[35] Q. Wang, T. R. Chan, R. Hilgraf, V. V. Fokin, K. B. Sharpless, M. G. Finn, J. Am. Chem. Soc. 2003, 125, 3192.
[36] J. E. Moses, A. D. Moorehouse, Chem. Soc. Rev. 2007, $36,1249$.

[37] A. H. El-Sagheer, T. Brown, Chem. Soc. Rev. 2010, 39, 1388

[38] C. E. Hoyle, C. N. Bowman, Angew. Chem. Int. Ed. 2010, 49, 1540.

[39] W. K. Lee, Z. T. Dai, W. P. King, P. E. Sheehan, Nano Lett. 2010, 10, 129.

[40] H. Y. Lin, L. C. Tsai, C. D. Chen, Adv. Funct. Mat. 2007, 17, 3182.

[41] Y. Cai, B. M. Z. Newby, J. Am. Chem. Soc. 2008, 130, 6076.

[42] Y. Elkasabi, H. Nandivada, H. Y. Chen, S. Bhaskar, J. D’Arcy, L. Bondarenko, J. Lahann, Chem. Vapor. Depos. 2009, 15, 142.

[43] H. Nandivada, H. Y. Chen, J. Lahann, Macromol. Rapid Comm. 2005, 26, 1794.

Received: September 25, 2010

Published online: February 8, 2011 